\title{
Externally controlled Lotka-Volterra dynamics in a linearly polarized polariton fluid
}

\author{
Matthias Pukrop ${ }^{1}{ }^{1}$ and Stefan Schumacher ${ }^{1,2}$ \\ ${ }^{1}$ Department of Physics and CeOPP, Universität Paderborn, Warburger Straße 100, 33098 Paderborn, Germany \\ ${ }^{2}$ College of Optical Sciences, University of Arizona, Tucson, Arizona 85721, USA
}

(Received 15 July 2019; published 13 January 2020)

\begin{abstract}
Spontaneous formation of transverse patterns is ubiquitous in nonlinear dynamical systems of all kinds. An aspect of particular interest is the active control of such patterns. In nonlinear optical systems this can be used for all-optical switching with transistorlike performance, for example, realized with polaritons in a planar quantum-well semiconductor microcavity. Here we focus on a specific configuration which takes advantage of the intricate polarization dependencies in the interacting optically driven polariton system. Besides detailed numerical simulations of the coupled light-field exciton dynamics, in the present paper we focus on the derivation of a simplified population competition model giving detailed insight into the underlying mechanisms from a nonlinear dynamical systems perspective. We show that such a model takes the form of a generalized Lotka-Volterra system for two competing populations explicitly including a source term that enables external control. We present a comprehensive analysis of both the existence and stability of stationary states in the parameter space spanned by spatial anisotropy and external control strength. We also construct phase boundaries in nontrivial regions and characterize emerging bifurcations. The population competition model reproduces all key features of the switching observed in full numerical simulations of the rather complex semiconductor system and at the same time is simple enough for a fully analytical understanding.
\end{abstract}

DOI: 10.1103/PhysRevE.101.012207

\section{INTRODUCTION}

The demand for integrated optoelectronic devices in optical communication networks has resulted in an increase of research activities targeted at functional all-optical components. For example, a wide range of different approaches has been proposed to realize efficient all-optical switches exploiting the nonlinear optical properties of different material systems, including organic photonic crystals [1], rubidium atomicvapor cells [2], and GaAs semiconductor microcavities as in Refs. [3-8]. The latter utilize the optical control of transverse optical patterns to achieve transistorlike switching performance [2,9]. Spontaneous formation of spatially extended patterns has been intensively studied during the past decades [10] with applications to different areas of science, including the formation of sand ripples and desert sand dunes [11], animal coat patterns such as zebra stripes [12,13], and geographic patterns in parasitic insect populations [14]. With applications to optical switching, however, the quest for efficient external control of these patterns naturally arises but in many cases has not been explored in detail. In the present work we investigate a specific example of all-optical switching of polariton patterns in a semiconductor quantum-well microcavity system. In contrast to our previous work [9] we give a detailed analysis of the optical switching dynamics from a nonlinear dynamical systems perspective. To this end we derive a simplified mode competition model that governs the essentials of the system dynamics but is simple enough to fully characterize the possible stationary solutions and phase-space singularities in the relevant parameter space. This simplified model has the very general mathematical form of a generalized Lotka-Volterra system, with the addition of an inhomogeneity for external control. In the main part of the paper (see Sec. III), we present a complete steady-state analysis of this nonlinear system. The solution space we obtain in dependence of spatial anisotropy and external control strength has a very general nature and may be similarly realized in other systems where external control of population competition is studied such as chemical reactions and in the life sciences.

As our specific example, here we study planar semiconductor microcavities with strong coupling between the cavity field and the exciton polarization that gives rise to the formation of exciton polaritons [15]. These quasiparticles consist of a photonic part and an excitonic part and are characterized by a normal-mode splitting in the dispersion relation, leading to long coherence times and strong nonlinear interactions on the lower branch. The latter are driven by four-wave mixing processes of coherent polariton fields which can also be interpreted as polariton-polariton scattering, mediated through exciton-exciton scattering. For certain excitation conditions, small spatially varying density fluctuations can experience huge growth in particular modes due to the intrinsic feedback mechanisms driven by four-wave mixing. This causes spatially homogeneous density distributions to become unstable such that the system's symmetry is spontaneously broken. This results in the formation of stationary patterns, directly observable in the far-field emission from the microcavity. Figure 1(a) shows the excitation geometry used with the pump at normal incidence (zero in-plane momentum) and finite offaxis $(k \neq 0)$ signals due to amplified fluctuations. Figure 1(b) shows the normal-mode splitting of the dispersion relation into lower and upper polariton branches alongside the bare 


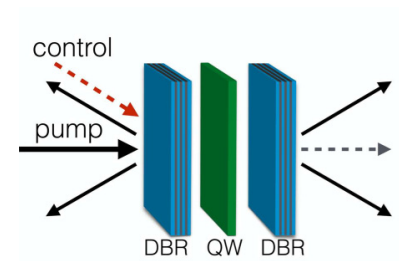

(a)

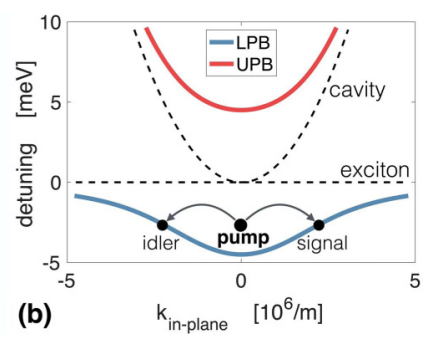

(b)

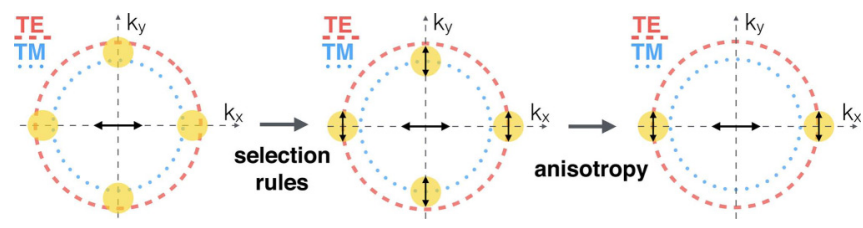

FIG. 2. Schematic illustration of the dominant contributions to the in-plane scattering of polaritons onto the elastic circles defined by the TE and TM modes of the lower polariton branch. The pump indicated in the center is $x$ polarized and slightly above the offaxis instability threshold. Parallel and perpendicular to the pump polarization direction, the scattering can occur in either the TE or TM mode (left panel). For pumping spectrally well below the exciton resonance, the scattering with polarization orthogonal to the pump's is preferred (middle panel). With spatial anisotropy (which is partly induced by the linear pump polarization) scattering along the pump's polarization direction onto the TE mode can dominate (right panel).

general, in any spatial direction signals can form either in the TE mode or in the TM mode, as illustrated in Fig. 2. However, for the pump polaritons scattered to finite $k$ through Coulomb interaction, due to the underlying spin-dependent excitonexciton interactions, the scattering probability is higher for a polarization state orthogonal to the pump polarization state [21]. Therefore, in the spatial direction parallel to the pump polarization state, the scattered signals preferentially form in the TE mode, and they form in the TM mode for scattering orthogonal to the pump polarization state (see Fig. 2). Out of these four signals, a stationary two-spot pattern (only one mode pair with opposite in-plane momenta) can be prepared by introducing some anisotropy favoring one direction over the other. Alongside a slight polarization-induced spatial anisotropy that is due to a slightly higher density of states for the TE modes [20], an additional anisotropy can be introduced by tilting the pump beam slightly away from normal incidence (see Fig. 2). In the nonlinear system studied, at sufficiently high densities of the favored two-spot pattern, cross-saturation processes will lead to the extinction of the signals in the other orthogonal direction. This allows us to single out a two-spot pattern $T_{1}$ which will serve as the initial state in the switching process. Here we start with the two-spot pattern oriented in the $x$ direction (the direction of intrinsic anisotropy). This selection process is schematically visualized in Fig. 2 .

If now a weak (compared to the pump intensity) $y$ polarized control beam with an in-plane momentum on the elastic circle and spatially orthogonal to the initial pattern is applied, stimulated scattering leads to population revival of the corresponding two-spot pattern. Due to the crosssaturation effect, the initial pattern is destabilized and finally switched off completely while the orthogonal pattern reaches a steady state $T_{2}$ (see Fig. 3). After the control beam has been switched off again, the anisotropy leads to reemergence of the initial $T_{1}$ pattern, while the $T_{2}$ state vanishes again. If the control beam is so weak that complete switching may not be possible, the system will remain in a stationary four-spot pattern state $F$. Based on this scheme, in Ref. [9] transistorlike reversible switching was demonstrated, including a systematic study of switching times, minimum control power needed, and achievable gain. This was done by numerical simulations of the nonlinear set of equations of motion governing the 

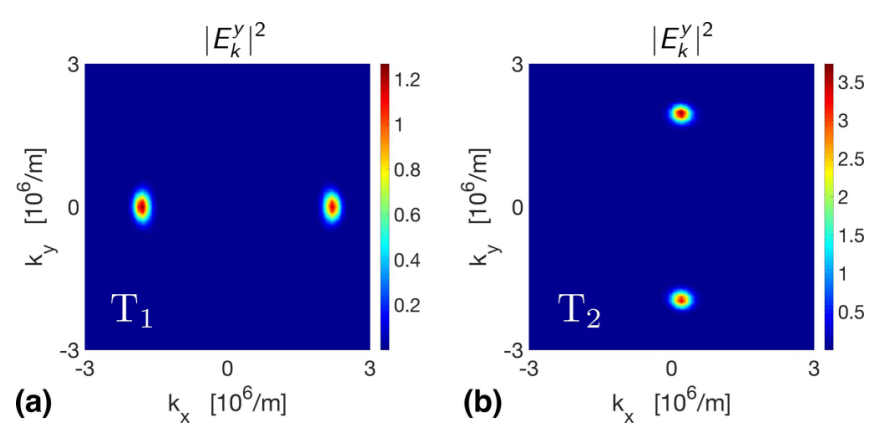

FIG. 3. (a) Stationary $T_{1}$ and (b) $T_{2}$ states of the polariton pattern switch as obtained from the numerical solution of Eqs. (1) for the $x$-linearly polarized $\mathrm{cw}$ pump at $k \approx 0$. Shown is the stationary $y$-polarization component of $|E|^{2}$ in $k$ space in arbitrary units (a) before and (b) after switching on a continuous-wave control beam. Switching off the control beam results in reversal of the switching process such that after sufficiently long times the system returns to the stationary $T_{1}$ pattern.

coherent coupled light field and exciton dynamics in the microcavity system in the two-dimensional QW plane in real space,

$$
\begin{aligned}
i \hbar \partial_{t} E^{ \pm}= & \left(H_{c}-i \gamma_{c}\right) E^{ \pm}+H^{ \pm} E^{\mp}-\Omega p^{ \pm}+E_{\mathrm{pump}}^{ \pm} \\
i \hbar \partial_{t} p^{ \pm}= & \left(\epsilon_{0}^{e}-i \gamma_{e}\right) p^{ \pm}-\Omega\left(1-\alpha_{\mathrm{psf}}\left|p^{ \pm}\right|^{2}\right) E^{ \pm} \\
& +T^{++}\left|p^{ \pm}\right|^{2} p^{ \pm}+T^{+-}\left|p^{\mp}\right|^{2} p^{ \pm}
\end{aligned}
$$

Here the index \pm denotes the different components in the circular polarization basis, $H_{c}=\hbar \omega_{0}-\frac{\hbar^{2}}{4}\left(\frac{1}{m_{\mathrm{TM}}}+\frac{1}{m_{\mathrm{TE}}}\right)\left(\partial_{x}^{2}+\partial_{y}^{2}\right)$ is the cavity Hamiltonian, and $\epsilon_{0}^{e}$ is the flat exciton dispersion. $\gamma_{c}$ and $\gamma_{e}$ represent the photon and exciton loss rates, $\Omega$ describes the photon-exciton coupling, and $H^{ \pm}=$ $-\frac{\hbar^{2}}{4}\left(\frac{1}{m_{\mathrm{TM}}}-\frac{1}{m_{\mathrm{TE}}}\right)\left(\partial_{x} \mp i \partial_{y}\right)^{2}$ couples the two polarization components due to TE/TM splitting. The cubic nonlinearities consist of a phase-space filling term $\alpha_{\mathrm{psf}}$, repulsive interaction $T^{++}$for excitons with parallel spins, and attractive interaction $T^{+-}$for excitons with opposite spins.

Based on Eqs. (1) with an $x$-linearly polarized continuouswave pump beam at $k \approx 0$, we perform a numerical simulation to demonstrate the basic switching process in the two-dimensional plane of in-plane momenta. Figure 3 shows the photonic component $|E|^{2}$ in $k$ space without a control beam such that the stationary pattern $T_{1}$ forms in Fig. 3(a) and, with the control beam on, switching to the stationary pattern $T_{2}$ in Fig. 3(b). The control beam has the same frequency as the pump beam (see Fig. 1). System parameters used and details of the calculations are given in Appendix A. Upon switching off the control beam the switching action is reversed, and the pattern returns to its original state $T_{1}$. Switching times in the range of $\sim 100 \mathrm{ps}$ are achievable [9].

The focus of the present paper is not on the full numerical simulations of Eqs. (1) and resulting switching performance. Rather, starting from Eqs. (1), we will derive a simplified population competition model for selected modes in $k$ space (details of the derivation are given in Appendix B) to provide further insight into the underlying phase space singularities that dictate the global behavior and solution space of the nonlinear dynamical system studied. In a similar fashion this approach was previously applied to the switching between subsets of a hexagonal pattern [22]. We will systematically analyze the existence and stability properties of possible steady states in dependence of the strength of the different involved physical processes for a typical orthogonal switching setup comparable to the one introduced above. To this end, we will construct phase boundaries in representative regions of parameter space and characterize relevant bifurcations. This will lead to a general understanding of crucial parameter dependencies such as the ratio between the control beam strength and the anisotropy and also of the coexistence of solutions in certain parameter regions and hysteresis behavior. Based on the simplified model, we will also be able to show that the polariton dynamics and optical switching phenomena discussed in the present paper can mathematically be understood based on a generalized Lotka-Volterra model including an external control parameter.

\section{POPULATION COMPETITION MODEL}

The simplified population competition model discussed in the remainder of the present paper can be derived from the set of equations of motion in Eq. (1) as detailed in Appendix B. It reads

$$
\begin{aligned}
& \dot{A_{1}}=\alpha_{1} A_{1}-\beta_{1} A_{1}^{3}-\theta_{1} A_{2}^{2} A_{1}, \\
& \dot{A_{2}}=\alpha_{2} A_{2}-\beta_{2} A_{2}^{3}-\theta_{2} A_{1}^{2} A_{2}+S .
\end{aligned}
$$

Here $A_{1}$ and $A_{2}$ are real-valued amplitudes of the two elementary states of the system, i.e., the two orthogonal polariton mode pairs in $k$ space. In the case of stationary solutions the phases in the full model (1) become "locked," allowing us to remove them as dynamical variables from the equations, which is suitable for the following steady-state analysis. The six dimensionless real-valued positive parameters, $\alpha_{i}$, $\beta_{i}, \theta_{i}$, are directly related to the main (up to third order) polariton-polariton scattering processes of the original system as illustrated in Fig. 4. They can be calculated from the physical parameters of the full model (see Appendix C). These parameters are intrinsically different for $A_{1}$ and $A_{2}$ due to the polarization dependence and anisotropy. The linear process representing growth of the resonant modes is described by $\alpha_{i}$. Saturation processes are represented by the cubic terms which can be divided into self-saturation $\beta_{i}$ and cross saturation $\theta_{i}$. The external control is described by the inhomogeneity $S$. If we rewrite Eqs. (2) as $\left(\dot{A}_{1}, \dot{A}_{2}\right)^{\mathrm{T}} \equiv\left(f_{1}, f_{2}\right)^{\mathrm{T}}=\mathbf{f}$, steady states are characterized by $\mathbf{f}=\mathbf{0}$. Four qualitatively different stationary solutions are possible: (i) two-spot pattern $T_{1}$ with $A_{1} \neq 0$ and $A_{2}=0$, (ii) two-spot pattern $T_{2}$ with $A_{1}=0$ and $A_{2} \neq 0$, (iii) four-spot pattern $F$ with $A_{1} \neq 0$ and $A_{2} \neq 0$, and (iv) the trivial solution with $A_{1}=0$ and $A_{2}=0$. Here we are interested in only physical solutions $A_{i} \geqslant 0$, and therefore, the state space is $\mathbb{R}_{\geqslant 0}^{2}$, i.e., the first quadrant of the $\left(A_{1}, A_{2}\right)$ plane. The linear term $\alpha$ leads to exponential growth of the corresponding mode pair, and the self-saturation $\beta$ has a stabilizing effect. For these two processes, Eqs. (2) are automatically decoupled and may be solved separately, resulting in a stable four-spot pattern. However, the cross-saturation terms $\theta$ couple the two modes and tend to suppress a particular mode pair, favoring the other mode pair, resulting in a two-spot 


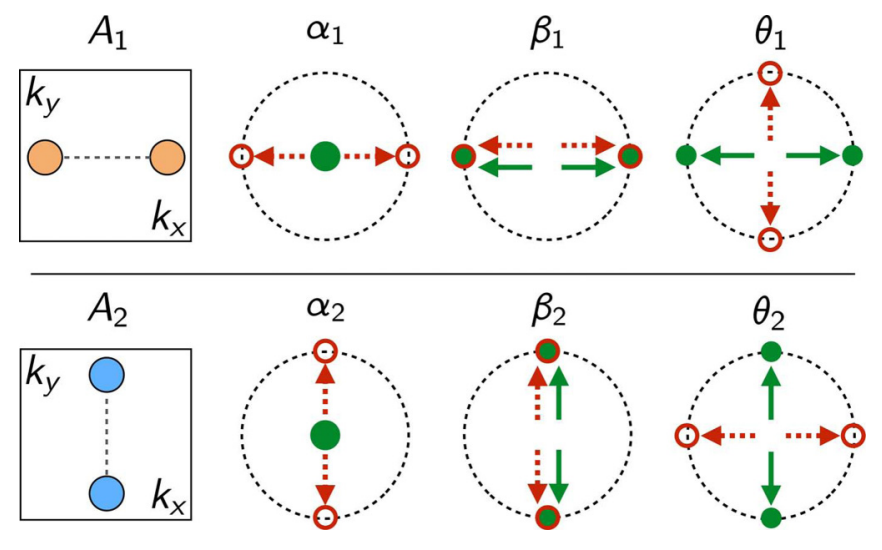

FIG. 4. Illustration of the different polariton-polariton scattering processes in $k$ space for the two elementary mode pairs $A_{1}$ and $A_{2}$ in Eq. (2). The dashed circle represents the ring in $k$ space on which efficient scattering is possible. Green solid (red open) circles mark the incoming (outgoing) modes. Solid (dashed) arrows mark their corresponding momenta. Here $\alpha_{i}$ describe the growth process of the two mode pairs (basic instability leading to pattern formation), and $\beta_{i}$ and $\theta_{i}$ represent the self- and cross-saturation processes.

pattern. Additionally, the external control acts as a source term for $A_{2}$. The PC model thus describes the dynamical competition between the three types of possible stationary patterns: $T_{1}, T_{2}$ and $F$. A phase in the PC model is defined as a set consisting of the number of steady states and their stability properties. These sets, i.e., the phase, are functions of the seven parameters, and a phase boundary in parameter space indicates the change in the number of steady states and/or their stability. This can happen only at points where at least one eigenvalue of the corresponding Jacobian matrix $J \equiv\left(\partial_{A_{j}} f_{i}\right)$ is zero [23], which is equivalent to the condition $\operatorname{det} J=0$. We thus find phase boundaries in parameter space at points satisfying $\{\mathbf{f}, \operatorname{det} J\}=\mathbf{0}$.

\section{A. Homogeneous case $S=0$}

The homogeneous case of Eq. (2) $(S=0)$ has the form of a generalized Lotka-Volterra (GLV) model [24] with cubic nonlinearities. The transformation $A_{i} \rightarrow A_{i}^{2} \equiv \widetilde{A}_{i}$ yields the usual Lotka-Volterra (LV) equations [25] with quadratic nonlinearities while conserving the $\left(A_{1}, A_{2}\right)$ phase space structure in the positive quadrant [26]. Hence, for $S=0$ the phase portraits of (2) are topologically equivalent to those of the following well-known system:

$$
\partial_{t} \tilde{A}_{i}=\tilde{A}_{i}\left(r_{i}-\sum_{j=1}^{2} c_{i j} \tilde{A}_{j}\right),
$$

with $i=1,2$, growth rate vector $\mathbf{r}=2\left(\alpha_{1}, \alpha_{2}\right)$, and community matrix

$$
C=2\left(\begin{array}{ll}
\beta_{1} & \theta_{1} \\
\theta_{2} & \beta_{2}
\end{array}\right),
$$

which describes self- and cross interaction. This LV model (3) for interspecific competition has been studied in many different contexts, e.g., ecology [27], chemistry [28], economics [29], and physics [30], and it was shown [25,31] that its dynamical behavior is limited to three cases depending on the parameters: (i) the coexistence regime for $\operatorname{sgn}(\operatorname{det} C)=+1$ (larger self-saturation), (ii) the bistability regime for $\operatorname{sgn}(\operatorname{det} C)=-1$ (larger cross saturation), and (iii) the dominance regime for sufficiently large and small growth rate ratios. In this regime long-time dynamics are independent of the interaction parameters and always result in extinction of one population. Although the LV system, Eq. (3), is well known, we present a short discussion of the homogeneous case of the PC model, Eq. (2), and point out the importance for application to the polariton switching dynamics. Since for $S=0$ the system is solvable analytically, we obtain explicit expressions for all steady states and phase boundaries: (i) for $T_{1}, A_{1}=\sqrt{\frac{\alpha_{1}}{\beta_{1}}}, A_{2}=0$, and it is stable for $\frac{\alpha_{1}}{\alpha_{2}}>\frac{\beta_{1}}{\theta_{2}}$, (ii) for $T_{2}, A_{1}=0, A_{2}=\sqrt{\frac{\alpha_{2}}{\beta_{2}}}$, and it is stable for $\frac{\alpha_{1}}{\alpha_{2}}<\frac{\theta_{1}}{\beta_{2}}$, (iii) for $F, A_{1}=\sqrt{\frac{\alpha_{2} \theta_{1}-\alpha_{1} \beta_{2}}{\theta_{1} \theta_{2}-\beta_{1} \beta_{2}}}, A_{2}=\sqrt{\frac{\alpha_{1} \theta_{2}-\alpha_{2} \beta_{1}}{\theta_{1} \theta_{2}-\beta_{1} \beta_{2}}}$, and it exists only for $\frac{\beta_{1}}{\theta_{2}} \lessgtr \frac{\alpha_{1}}{\alpha_{2}} \lessgtr \frac{\theta_{1}}{\beta_{2}}$ and is stable for $\theta_{1} \theta_{2}<\beta_{1} \beta_{2}$, and (iv) for the trivial solution, $A_{1}=A_{2}=0$. We already see that the four-spot solution does not exist in the entire $S=0$ plane, in contrast to the two-spot pattern. The trivial solution also exists everywhere but is always unstable since the eigenvalues of its Jacobian $\alpha_{1}, \alpha_{2}$ are positive. This solution will not be listed hereinafter. For a systematic discussion we introduce a variable anisotropy parameter $\delta \alpha$ in the first equation of (2), $\alpha_{1} \rightarrow \alpha_{1}+\delta \alpha$, and set $\alpha_{1}=\alpha_{2}=1$. It favors $A_{1 / 2}$ for $\delta \alpha \gtrless 0$, respectively. The resulting phase boundaries are shown in Fig. 5, where the homogeneous case $(S=0)$ is included in the extended region at the bottom of each plot. It shows the structure of the usual LV model. Green (dark gray) and red (light gray) letters mark stable and unstable steady states, respectively. For large anisotropy $|\delta \alpha|$ only stable and unstable two-spot patterns are possible. This dominance regime no longer depends on the interaction parameters $\beta_{i}$ and $\theta_{i}$. The middle region, on the other hand, is divided into two cases: a stable four-spot pattern for larger self-saturation [ $\beta_{1} \beta_{2}>\theta_{1} \theta_{2}$, coexistence, Fig. 5(a)] and two simultaneously stable two-spot patterns for larger cross saturation $\left[\beta_{1} \beta_{2}<\theta_{1} \theta_{2}\right.$, bistability, Fig. 5(b)].

The first four cases in Fig. 6 show the corresponding flow given by representative trajectories in state space for the homogeneous case. The system's dynamics are unambiguous in cases 1,2 , and 3a, where only one attractor exists which determines the long-time behavior. However, in case $3 b$ two attractors exist, and the dynamics now depend on the system's history, leading to a hysteresis effect. The two basins of attraction are marked with different colors, blue (dark gray) and orange (light gray). They are separated by the stable manifold of the saddle point $F$ (often called the separatrix), defined by the set of points $\left(A_{1}, A_{2}\right)$ which satisfy $\left(A_{1}, A_{2}\right) \rightarrow F$ for $t \rightarrow \infty$. In contrast to this, the line connecting all three steady states is the unstable manifold of $F$, consisting of points which satisfy $\left(A_{1}, A_{2}\right) \rightarrow F$ for $t \rightarrow-\infty$. Any initial point will stay in its region (blue or orange) and end up at the corresponding attractor $\left(T_{1}\right.$ or $\left.T_{2}\right)$. The system thus shows hysteresis behavior which might prevent complete back switching and therefore should be avoided for switching purposes, for example, by increasing the anisotropy. 
(a)

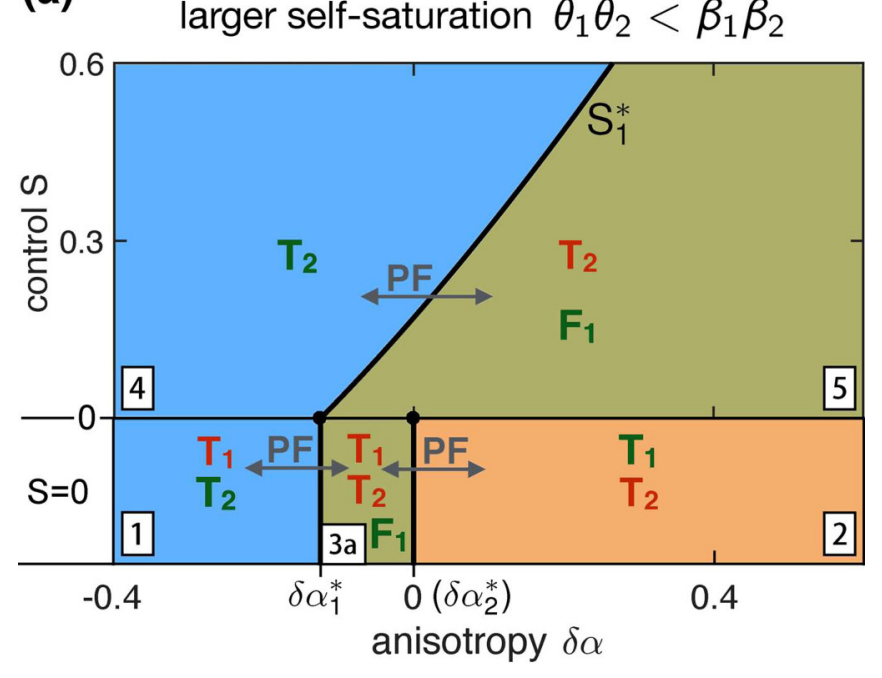

(b)

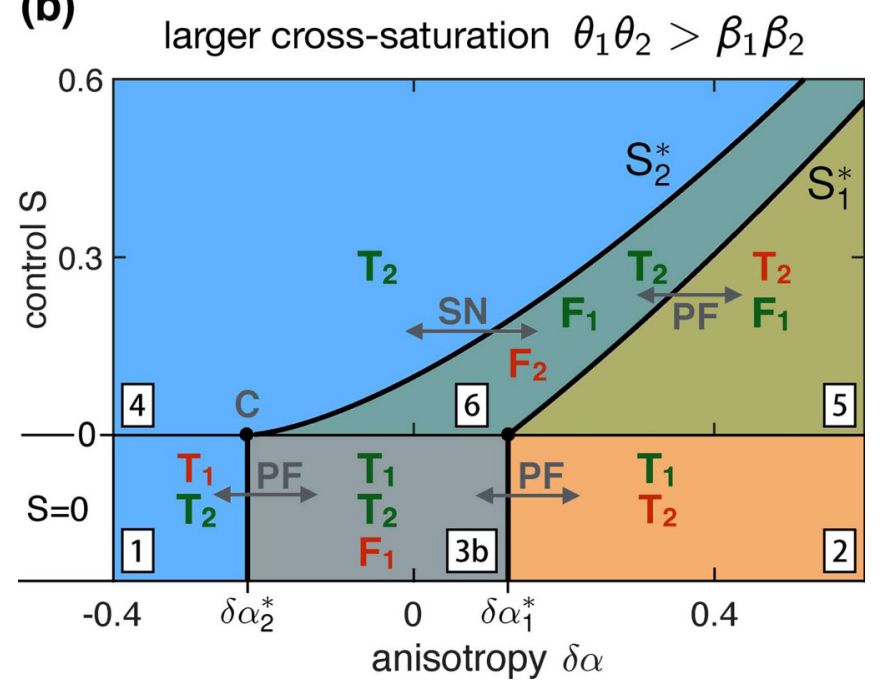

FIG. 5. Phase boundaries in $(\delta \alpha, S)$ parameter space showing regions of different stable and unstable steady states in dependence of the anisotropy and external control. (a) Larger self-saturation $\beta_{1} \beta_{2}>\theta_{1} \theta_{2}$. (b) Larger cross saturation $\beta_{1} \beta_{2}<\theta_{1} \theta_{2}$. Green (dark gray) and red (light gray) letters mark stable and unstable steady states, respectively. Lines $S_{1}^{*}$ and $S_{2}^{*}$ show the phase boundaries (5) and (6).

The Hartman-Grobman theorem [23] ensures that the behavior near hyperbolic equilibrium points is completely determined by its linearization. This no longer holds for nonhyperbolic fixed points which are characterized by the existence of at least one zero real-part eigenvalue of the corresponding Jacobian. Therefore, the behavior at the phase boundaries in parameter space cannot be analyzed via linear stability analysis. Instead one can use center manifold theory and normal forms [23] in order to determine the occurring bifurcations. They are transcritical for the usual LV model but different in the case of cubic nonlinearities due to the additional $\mathbb{Z}_{2} \times \mathbb{Z}_{2}$ symmetry. For both two-spot solutions symmetric pitchfork (PF) bifurcations occur at the phase boundaries when the anisotropy parameter is changed. They are supercritical for $\beta_{1} \beta_{2}>\theta_{1} \theta_{2}$ (larger self-saturation), leading to the coexistence regime with a stable $F$ pattern, and subcritical for $\beta_{1} \beta_{2}<\theta_{1} \theta_{2}$
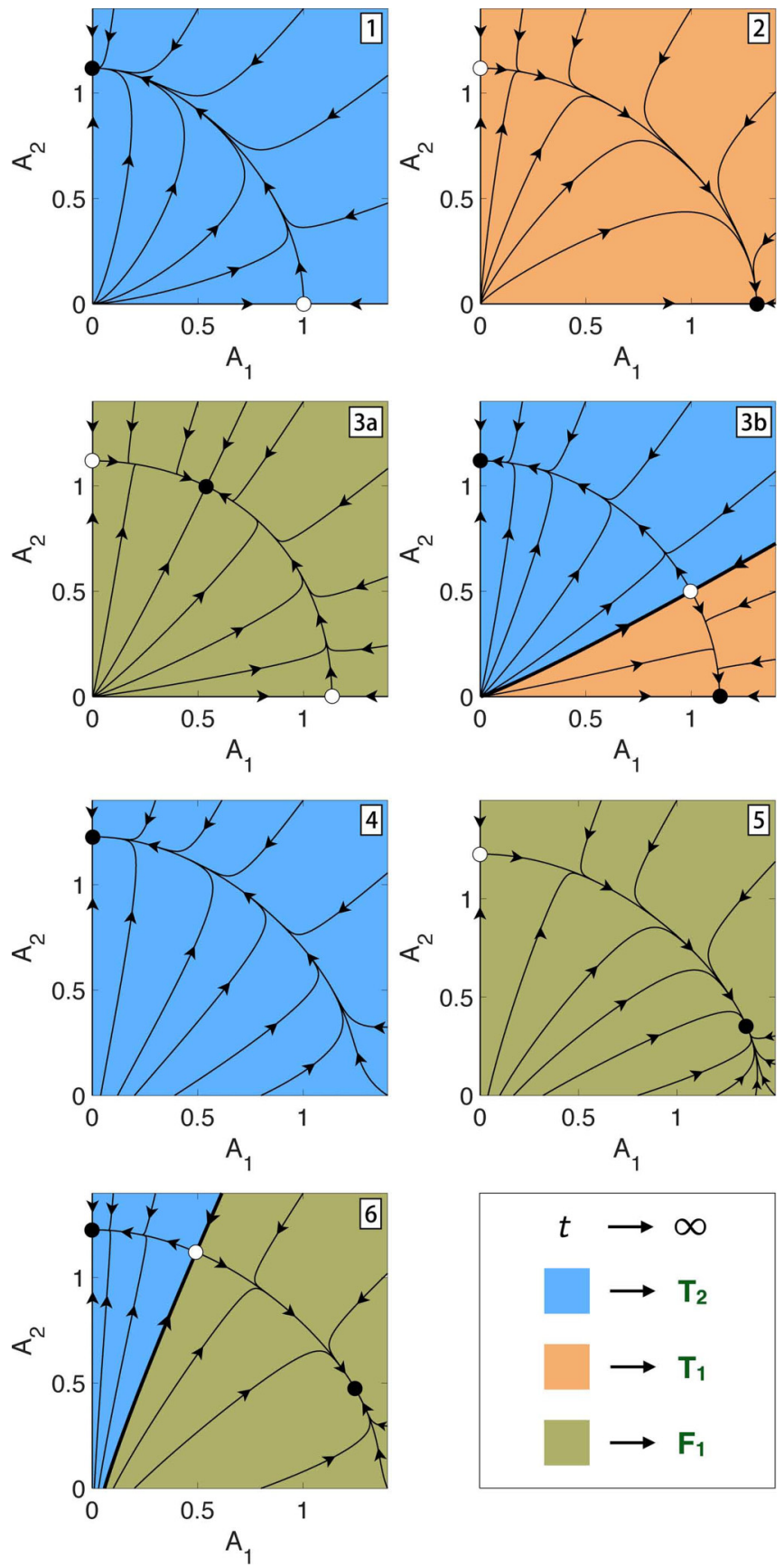

FIG. 6. Phase plane flow for cases corresponding to Fig. 5. Black (white) dots mark stable (unstable) steady states. Black lines show representative orbits. The thick black line in plots $3 b$ and 6 is the stable manifold of the saddle point which separates two basins of attraction.

(larger cross saturation), leading to the bistability regime with a stable $T_{1}$ and $T_{2}$ pattern. Pitchfork bifurcations are typical for dynamical systems with inversion symmetry, here $A_{i} \rightarrow-A_{i}$. Since we are interested in only positive solutions in the first quadrant of the phase plane, we observe either a transition from a stable $T$ solution to an unstable $T$ and stable $F$ solution (supercritical) or the same transition with reversed stability (subcritical).

In conclusion, all phase portraits of the homogeneous case can be completely reduced to the results of the well-known 
Lotka-Volterra model for two competitive species. Due to the additional inversion symmetry we obtain pitchfork bifurcations at the phase boundaries. The homogeneous case is crucial for the polariton switch because it describes the initial pattern formation and back-switching process in the absence of the control beam. Both can work reliably only in regions where only the $T_{1}$ pattern exists as a single attractor. Therefore, a sufficient minimum anisotropy in favor of $A_{1}$ is needed. We have also seen that if the cross saturation dominates over the self-saturation, the coexistence of $A_{1}$ and $A_{2}$ is destabilized, resulting in the extinction of $A_{i}$ and survival of $A_{i+1}$. A strong interspecific competition thus prevents the coexistence. Otherwise, if the self-saturation is stronger than the cross saturation, the dominating intraspecific competition promotes coexistence. The parameters used for the full numerical simulations in Sec. II lead to the larger self-saturation case and sufficient anisotropy (see Appendix C), providing reliable initial pattern formation and back switching since the $F$ pattern is unstable.

\section{B. Inhomogeneous case $S>0$}

The inhomogeneous $(S>0)$ case of the PC model (2) describes the actual switching process induced by an external control beam. This term can also be motivated for other systems where the GLV model is commonly used, e.g., to include constant migration and harvesting in the description of ecological systems or constant influx in chemical reactions. Therefore, it can be interpreted as an extension of the generalized Lotka-Volterra dynamics, and the following analysis is of very general interest but has not been investigated before. The influence of constant terms in the usual LV model with quadratic nonlinearities was investigated in Ref. [32] from a purely mathematical perspective, but in general inhomogeneous population competition models have not received much attention in the past. Here we analyze and apply the GLV model with a constant inhomogeneity to the case of the orthogonal switching of two-spot polariton patterns.

Also including inhomogeneity, all steady states and phase boundaries can still be determined analytically due to the system's simplicity. However, we note that, in general, solving multivariate polynomial equation systems is a difficult task which can be simplified using algebraic methods (e.g., Gröbner's basis [33,34]), as was shown in Ref. [22]. The nonzero source term $S$ in (2) breaks the inversion symmetry for $A_{2}$ and prevents a $T_{1}$ solution. This leaves us with only two competing patterns (called $T_{2}$ and $F_{2}$ ) this time around. However, as we will see below, it includes the possibility of an additional qualitatively different four-spot pattern solution $F_{1}$. The control parameter $S$ acts as a constant source term for the $A_{2}$ mode pair, leading to linear growth. Supporting $A_{2}$ means simultaneously suppressing $A_{1}$ due to the cross-saturation effect, leading to a more asymmetric $F_{2}$ solution in favor of $A_{2}$. A second four-spot pattern, $F_{1}$, which is in favor of $A_{1}$, replaces $T_{1}$. The phase boundaries are given explicitly by

$$
S_{1}^{*}(\delta \alpha)=\beta_{2}{\sqrt{\frac{1+\delta \alpha}{\theta_{1}}}}^{3}-\sqrt{\frac{1+\delta \alpha}{\theta_{1}}},
$$

and additionally, for the case of larger cross saturation $\left(\beta_{1} \beta_{2}<\theta_{1} \theta_{2}\right)$

$$
S_{2}^{*}(\delta \alpha)=6 \sqrt{\frac{3\left[(1+\delta \alpha) \theta_{2}-\beta_{1}\right]^{3}}{\beta_{1}^{2}\left(\theta_{1} \theta_{2}-\beta_{1} \beta_{2}\right)}} .
$$

The explicit expressions for the steady states $T_{2}, F_{1}$, and $F_{2}$ are not given here due to their excessive length. The phase boundaries in the $(\delta \alpha, S)$ parameter space can be seen in Fig. 5 for the cases of both higher self-saturation and higher cross saturation. Starting from the threshold values $\delta \alpha_{1,2}^{*}$ of the homogeneous case, we find continuously changing phase boundaries $S_{1,2}^{*}$ to higher anisotropy values for increasing control $S$. In the case of higher self-saturation there is only one phase boundary $S_{1}^{*}$ due to the absence of a $T_{1}$ state. For sufficiently strong control $S$ there is only the stable steady state $T_{2}$. This region represents the desired outcome of a successful switching process. Its phase boundary determines the minimum control strength required to achieve switching for a given anisotropy value. For lower values of $S$, a stable four-spot pattern arises, and depending on the ratio $\frac{\theta_{1} \theta_{2}}{\beta_{1} \beta_{2}}$, a second unstable four-spot pattern occurs together with a stability transition of $T_{2}$.

Again, we can draw the flow in the $\left(A_{1}, A_{2}\right)$ phase plane and mark the basins of attraction as shown in Fig. 6, cases 4 , 5 , and 6. For $\theta_{1} \theta_{2}>\beta_{1} \beta_{2}$ a region with two attractors occurs similar to the $S=0$ case, but this time with a stable $F_{1}$ state instead of $T_{1}$, which again implies hysteresis behavior. For increasing anisotropy, the unstable $F_{2}$ state will approach $T_{2}$ along the unstable manifold until they meet and an unstable $T_{2}$ state and a stable $F_{1}$ state emerge. This corresponds to the approach of the two phase boundaries $S_{1}^{*}$ and $S_{2}^{*}$ and the annihilation of $S_{2}^{*}$, which can happen only for parameter values $1<\frac{\theta_{1} \theta_{2}}{\beta_{1} \beta_{2}}<\frac{3}{2}$. Otherwise, the two boundaries diverge. Thus, the case of higher cross saturation is divided into two subcases, defined by either the survival or the vanishing of the middle region with two attractors.

Another important effect of the inhomogeneity $S$ is the explicit breaking of the $\mathbb{Z}_{2}$ symmetry in the time evolution of $A_{2}$. In the case of larger cross saturation a cusp point $C$ arises in the two-dimensional bifurcation diagram in Fig. 5(b). The pitchfork bifurcation at $S=0$ is replaced by a saddle-node bifurcation for $S \neq 0$, resulting in the creation or destruction of a stable-unstable pair of $F$ solutions while varying one of the parameters. In the cusp point two saddle-node phase boundaries, $S_{2}^{*}$ and $-S_{2}^{*}$ (not shown), meet tangentially. The other pitchfork bifurcation $\left(\delta \alpha_{1}^{*}\right)$ remains $\left(S_{1}^{*}\right)$ in the presence of the inhomogeneity since the $\mathbb{Z}_{2}$ symmetry of $A_{1}$ is still conserved. This is the only bifurcation in the case of larger self-saturation in Fig. 5(a) for $S \neq 0$. Figure 7 shows the evolution of the equilibrium surfaces for $A_{1}$ and $A_{2}$ in the $(\delta \alpha, S)$ parameter space and the corresponding bifurcations. The pitchfork bifurcations are visible on the $S=0$ line. In the case of higher cross saturation [Fig. 7(b)] one of the pitchfork bifurcations unfolds into a saddle-node bifurcation with increasing $S$. In the case of higher self-saturation [Fig. 7(a)] the supercritical pitchfork bifurcation remains stable with increasing $S$.

In conclusion, the main difference for the inhomogeneous case is the absence of a steady $T_{1}$ state and the existence of an 

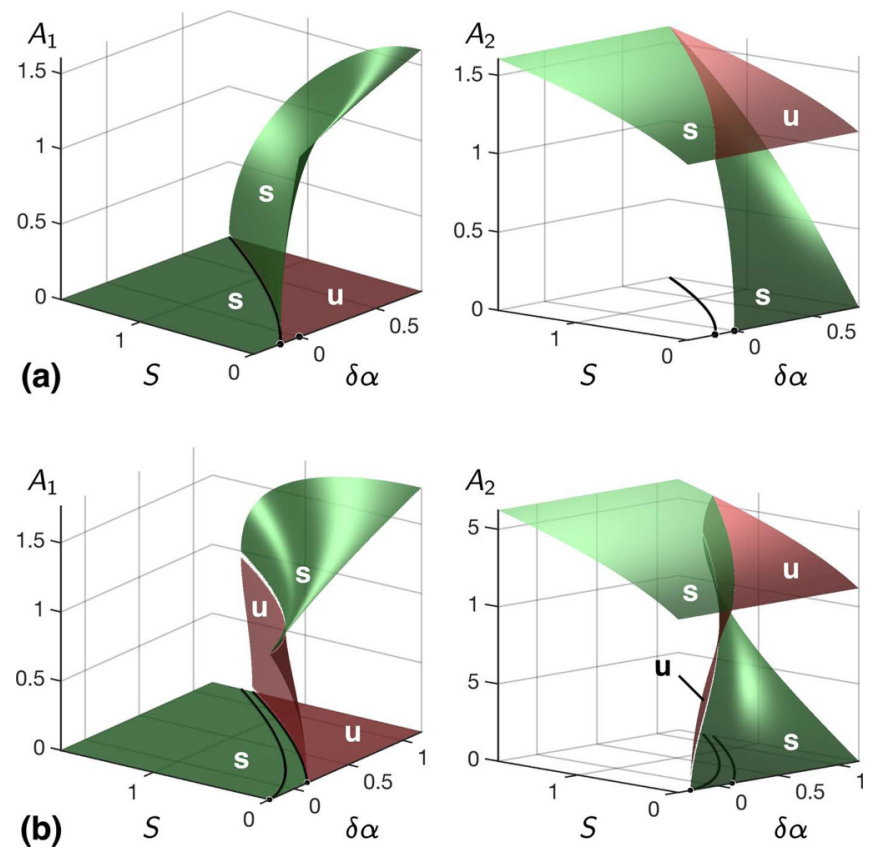

FIG. 7. Evolution of steady states for $A_{1}$ and $A_{2}$ in parameter space. Green (s) and red (u) surfaces belong to stable and unstable solutions, respectively. Black lines correspond to the phase boundaries projected on the $(\delta \alpha, S)$ plane. (a) Larger self-saturation: one of the two pitchfork bifurcations on the $S=0$ line remains for $S>0$, whereas the other one vanishes completely. (b) Larger cross saturation: one of the two pitchfork bifurcations on the $S=0$ line remains for $S>0$; the other one is replaced by a saddle-node bifurcation.

additional stable $F_{1}$ state in the case of higher cross saturation. This corresponds to the unfolding of one of the subcritical pitchfork bifurcations into a saddle-node bifurcation. The control term $S$ thus prevents extinction of $A_{2}$ and promotes coexistence. Furthermore, the $F_{1}-T_{2}$-bistability region vanishes for high anisotropy values if the parameter condition $1<\frac{\theta_{1} \theta_{2}}{\beta_{1} \beta_{2}}<\frac{3}{2}$ is satisfied, resulting in a single remaining phase boundary $S_{1}^{*}$ similar to the case of higher self-saturation. The occurring saddle-node and subcritical pitchfork bifurcations are problematic since they both imply sudden vanishing of a stable fixed point, meaning the system undergoes an abrupt transition to another stable fixed point; that is, hysteresis is possible. This can happen only in the bistability regime (larger cross saturation). The numerical simulation of the switching process presented in Sec. II takes place in the larger self-saturation regime (model parameters are calculated in Appendix C), which is advantageous for the switching purpose since no hysteresis can occur. However, if the external control beam is not sufficiently strong, we observe a stable $F_{1}$ pattern in the numerical simulations, as predicted by the PC model.

\section{Remarks}

So far, we have discussed steady states, their stability properties, and bifurcations occurring in the solution space of the population competition model in Eq. (2). From a dynamical perspective, we observe critical slowing down $[35,36]$ near the phase boundaries due to the continuously vanishing real part of the Jacobian eigenvalue responsible for the bifurcation. This corresponds to the divergence of switching times observed in the numerical simulations in Ref. [9]. For example, approaching $S_{1,2}^{*}$ for $\beta_{1} \beta_{2} \gtrless \theta_{1} \theta_{2}$ from above results in divergence of the switching time. Similarly, approaching $\delta \alpha_{2,1}^{*}$ for $\beta_{1} \beta_{2} \gtrless \theta_{1} \theta_{2}$ from the left side results in divergence of the back-switching time. Hence, to achieve favorable performance, switching should be done for parameters sufficiently far away from the phase boundaries.

We note that, in general, a nonlinear dynamical system can have periodic solutions which are characterized by closed orbits in the phase plane. Here we use Dulac's criterion [37] to rule out any periodic solutions. A simplified version reads as follows: The existence of a function $g\left(A_{1}, A_{2}\right)$ with the property that $\nabla \cdot(g \mathbf{f})$ is sign definite in the entire considered state space rules out any closed orbits in this area. If we choose $g=\frac{1}{A_{1} A_{2}}$, we obtain $\nabla \cdot(g \mathbf{f})=-2\left(\beta_{1} \frac{A_{1}}{A_{2}}+\beta_{2} \frac{A_{2}}{A_{1}}+\frac{S}{A_{1} A_{2}^{2}}\right)$, and therefore, closed orbits in the positive quadrant are impossible. Another observation is that for $\theta_{1}=\theta_{2} \equiv \theta$ (symmetric coupling) we can write the system in Eq. (2) as a gradient field $\mathbf{f}=\nabla V$ with the potential function

$$
V=\sum_{i=1}^{2} \frac{\alpha_{i}}{2} A_{i}^{2}-\frac{\beta_{i}}{4} A_{i}^{4}-\frac{\theta}{4} A_{i}^{2} A_{i+1}^{2}+S_{i} A_{i},
$$

with $A_{i+2}=A_{i}, S_{1}=0$, and $S_{2}=S$. Closed orbits are impossible in gradient systems [37]; however, for the general case $\theta_{1} \neq \theta_{2}$ (asymmetric coupling) this argument is no longer applicable. Also, assuming the gradient system defined by the potential function (7) allows us to use the language of catastrophe theory and observe that two of Thom's seven elementary catastrophes occur, namely, folds (denoted as $A_{2}$ in Arnold's notation) and cusps $\left(A_{3}\right)$. We further note that our detailed investigation above is limited to destabilizing linearity, stabilizing nonlinearities, and positive control parameter. These conditions match the numerical and experimental observations for the physical system under investigation here. For this case we are able to completely characterize all possible steady states and their corresponding bifurcations and rule out any other bifurcations involving double-zero and purely imaginary eigenvalues of the Jacobian.

\section{CONCLUSIONS}

We have presented a detailed analysis of an all-optical switching concept based on transverse patterns in an interacting polariton system in a planar quantum-well semiconductor microcavity. From the relevant equations based on a microscopic semiconductor theory here we derived a simplified population competition (PC) model describing the system dynamics restricted to selected modes in $k$ space. Interestingly, the resulting rather simple PC model shows all key features of the system dynamics also observed in the full numerical simulations in the parameter range of interest here. In addition to what can be learned from the numerical simulations, the PC model enables us to systematically identify phase boundaries in parameter space and singularities governing the global dynamics of the nonlinear system. Interestingly, the rather complicated original system of interacting microcavity 
polaritons for the switching phenomenon studied here can be completely characterized by only seven flow portraits in a two-dimensional state space. The model derived has the very generic form of a generalized Lotka-Volterra system extended with an inhomogeneity term to achieve external control. Such a system has not been investigated before. Considering the widespread use of GLV systems, the understanding obtained in the present work is of a very general nature and will be similarly applicable to other fields where external control of population competition is studied such as chemical reactions or population competition in the life sciences.

\section{ACKNOWLEDGMENTS}

We gratefully acknowledge funding from the Deutsche Forschungsgemeinschaft through project SCHU 1980/5-2 and through the Heisenberg program (Grant No. 270619725). We further acknowledge valuable discussions with R. Binder and a grant for computing time at the Paderborn Center for Parallel Computing $\left(\mathrm{PC}^{2}\right)$.

\section{APPENDIX A: NUMERICAL DETAILS}

We used the following parameters (appropriate for GaAs systems) to numerically simulate the switching presented in Sec. II: $m_{\mathrm{TE}}=1.05 m_{\mathrm{TM}}=0.215 \mathrm{meV} \mathrm{ps}^{2} \mu \mathrm{m}^{-2}$, $\Omega=6.5 \mathrm{meV}, \gamma_{c}=0.8 \mathrm{meV}, \gamma_{e}=0.2 \mathrm{meV}, \alpha_{\mathrm{psf}}=5.188 \times$

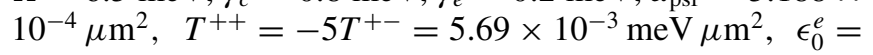
$1.497 \mathrm{eV}$. Coherent cw excitation $2.5 \mathrm{meV}$ above the lower polariton branch was used with flat-top Gaussian profiles with intensities $I_{\text {pump }}=2.1 \times 10^{5} \times I_{\text {probe }}=54 \mathrm{~kW} \mathrm{~cm}^{-2}$. Equations (1) were solved on a finite two-dimensional grid in real space using a fourth-order Runge-Kutta method with a variable step size. In Sec. III we use parameters $\beta_{1} \beta_{2} / \theta_{1} \theta_{2}=$ 1.14 for the case of larger self-saturation and $\beta_{1} \beta_{2} / \theta_{1} \theta_{2}=$ 0.69 for the case of larger cross saturation. All bifurcations were determined analytically for the homogeneous case and numerically with the help of MATCONT, a MATLAB software package, for the inhomogeneous case.

\section{APPENDIX B: DERIVATION OF THE PC MODEL}

We follow qualitatively the derivation of the hexagon PC model [22], but here we are including polarization effects for linearly polarized excitation and therefore considering a different reduced $k$ space. We start with the coupled equations of motion (1) for the exciton polarization and the cavity field and transform them into $k$ space and in the linear polarization basis. Introducing the reduced $k$ space consisting of modes $\left\{k_{0} \equiv 0, k_{1}, k_{2}, k_{3}, k_{4}\right\}$ with relations $k_{3}=-k_{1}$ and $k_{4}=-k_{2}$ (see Fig. 8) results in 20 equations. We consider an $x$-polarized pump. This leads to the following selection rules [19]: (i) the ${ }_{\mathrm{y}}^{\mathrm{x}}$-polarized probe with $\mathbf{k} \| \mathbf{e}_{x}$ excites ${ }_{\mathrm{TE}}^{\mathrm{TM}}$ mode, and (ii) the ${ }_{\mathrm{y}}^{\mathrm{x}}$-polarized probe with $\mathbf{k} \perp \mathbf{e}_{x}$ excites the ${ }_{T M}^{T E}$ mode.

We assume that all dynamical quantities oscillate with pump frequency $\omega$. Removing the phase factor $\mathrm{e}^{-i \omega t}$ yields a shift of the dispersion by $-\hbar \omega$. We consider all phase-matched scattering processes up to third order within the reduced

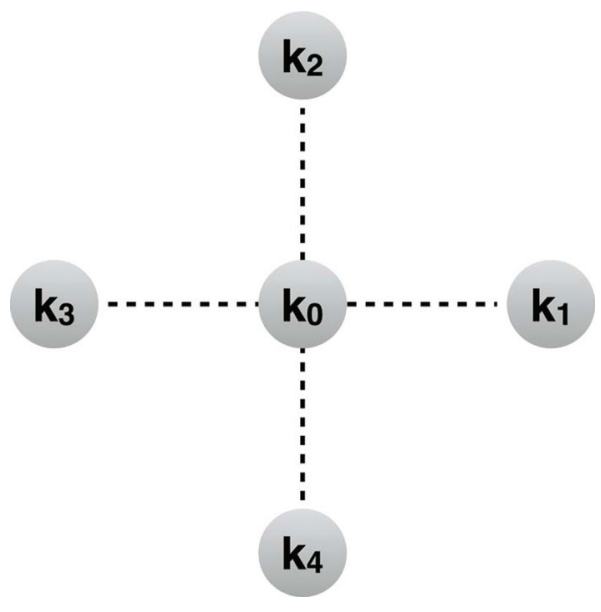

FIG. 8. Definition of the reduced $k$ space.

$k$ space. In agreement with the linear stability analysis of the corresponding system reporting a $D_{2} \cong \mathbb{Z}_{2} \times \mathbb{Z}_{2}$ symmetry [20], we assume an equal excitation of opposite modes in the reduced $k$ space, i.e., $p_{\mathbf{k}_{1}}=p_{\mathbf{k}_{3}} \equiv p_{1}$ and $p_{\mathbf{k}_{2}}=p_{\mathbf{k}_{4}} \equiv p_{2}$ (with analogous notation for $E$ ). We further assume the pumpinduced densities $E_{0}^{x / y}$ and $p_{0}^{x / y}$ at $k=0$ are constant. Using a cyclic definition for the two mode pairs $p_{j}=p_{j+2}$, with $j=1,2$, results in the following phase-matched $(\mathbf{q}=\mathbf{k}-$ $\left.\mathbf{k}^{\prime}-\mathbf{k}^{\prime \prime}\right)$ scattering processes $\left(\mathbf{q}, \mathbf{k}^{\prime}, \mathbf{k}^{\prime \prime}\right)$ for each mode pair in Eq. (1):

$$
\begin{aligned}
& (j, 0,0), \quad(0, j, 0), \quad(0,0, j), \\
& 3 \times(j, j, j), \quad 2 \times(j, j+1, j+1) .
\end{aligned}
$$

In our setup the pump is $x$ polarized. Therefore, terms $\propto p_{0}^{y}$ or $\propto E_{0}^{y}$ are omitted. Furthermore, we assume the colinearly polarized off-axis modes are very small as for these modes the instability threshold is not reached, i.e., $p_{j}^{x} \ll p_{j}^{y}$ and $E_{j}^{x} \ll E_{j}^{y}$. Hence, we also neglect terms $\propto p_{j}^{x}$ or $\propto E_{j}^{x}$. This leaves us with the following four equations for the $y$-polarized mode pairs:

$$
\begin{aligned}
i \hbar \partial_{t} p_{j}^{y}= & \left(\epsilon_{j}-\hbar \omega-i \gamma_{e}\right) p_{j}^{y}+\frac{1}{2} \alpha_{\mathrm{PSF}} \Omega\left[-p_{j}^{y *} p_{0}^{x} E_{0}^{x}+p_{0}^{x *} p_{j}^{y} E_{0}^{x}\right. \\
& \left.+\left|p_{0}^{x}\right|^{2} E_{j}^{y}+3 p_{j}^{y *} p_{j}^{y} E_{j}^{y}+2 p_{j}^{y *} p_{j+1}^{y} E_{j+1}^{y}\right] \\
& +\frac{1}{2}\left(T^{++}+T^{+-}\right)\left[3 p_{j}^{y *} p_{j}^{y} p_{j}^{y}+2 p_{j}^{y *} p_{j+1}^{y 2}\right] \\
& -\frac{1}{2}\left(T^{++}-T^{+-}\right)\left[p_{j}^{y *} p_{0}^{x 2}\right]+T^{++}\left|p_{0}^{x}\right|^{2} p_{j}^{y}-\Omega E_{j}^{y},
\end{aligned}
$$

$$
i \hbar \partial_{t} E_{j}^{y}=\left(\hbar \omega_{j}^{y}-\hbar \omega-i \gamma_{c}\right) E_{j}^{y}-\Omega p_{j}^{y}+E_{\text {pump }, j}^{y} .
$$

If we further assume that the time evolution of $E$ follows adiabatically the evolution of $p$ and set $\partial_{t} E_{j} \approx 0$, we can write

$$
E_{j}=\frac{\Omega}{\hbar \omega_{j}-\delta_{j}-\hbar \omega-i \gamma_{c}} p_{j} \equiv \frac{2 \lambda_{j}}{\alpha_{\mathrm{PSF}} \Omega} \mathrm{e}^{i \theta_{j}} p_{j},
$$

with $j=0,1,2$. The pump field was also set to zero $E_{\text {pump }, j}=0$ and will be added later manually. We define $\theta_{j}$ as 
the phase between $E_{j}^{y}$ and $p_{j}^{y}$, and the ratio of their amplitudes is given by $\frac{2 \lambda_{j}}{\alpha_{\mathrm{PSF}} \Omega}$. Here $\delta_{j}$ includes anisotropy effects due to the higher density of states for the TE modes and tilting of the pump. So the parameters are given by

$$
\begin{array}{cc}
\lambda_{j} e^{i \theta_{j}}=\frac{\alpha_{\mathrm{PSF}} \Omega^{2}}{2\left(\hbar \omega_{j}^{y}-\delta_{j}-\hbar \omega-i \gamma_{c}\right)} & \text { for } j=1,2, \\
\lambda_{0} e^{i \theta_{0}}=\frac{\alpha_{\mathrm{PSF}} \Omega^{2}}{2\left(\hbar \omega_{0}^{x}-\hbar \omega-i \gamma_{c}\right)} & \text { for } j=0 .
\end{array}
$$

Finally, we obtain two equations for the two elementary states of the system. We also factorize the exciton field into phase and magnitude, i.e., $p_{j}^{y}=\tilde{p}_{j}^{y} e^{i \varphi_{j}}$, and split the results into separate equations of motion for magnitude and phase:

$$
\begin{gathered}
\partial_{t} \tilde{p}_{j}^{y}=L_{j} \tilde{p}_{j}^{y}+\sum_{k=1}^{2} C_{j k} \tilde{p}_{k}^{y 2} \tilde{p}_{j}^{y}, \\
\partial_{t} \varphi_{j}=K_{j}+\sum_{k=1}^{2} D_{j k} \tilde{p}_{k}^{y 2} .
\end{gathered}
$$

If we define $\phi_{j} \equiv \varphi_{j}-\varphi_{0}$, the coefficients are then given by

$$
\begin{aligned}
\hbar L_{j} & =-\gamma_{e}+\lambda_{0} \tilde{p}_{o}^{x 2}\left[\sin \left(\theta_{0}\right)-\sin \left(\theta_{0}-2 \phi_{j}\right)\right]+\lambda_{j} \sin \left(\theta_{j}\right)\left(\tilde{p}_{0}^{x 2}-\frac{2}{\alpha_{\mathrm{PSF}}}\right)-\frac{1}{2}\left(T^{++}-T^{+-}\right) \tilde{p}_{0}^{x 2} \sin \left(-2 \phi_{j}\right), \\
\hbar C_{j k} & =\left\{\begin{array}{l}
3 \lambda_{j} \sin \left(\theta_{j}\right), \quad j=k, \\
2 \lambda_{k} \sin \left(\theta_{k}+2 \phi_{k}-2 \phi_{j}\right)+\left(T^{++}+T^{+-}\right) \sin \left(2 \phi_{k}-2 \phi_{j}\right), \quad j \neq k,
\end{array}\right. \\
\hbar K_{j} & =\epsilon_{j}-\hbar \omega-T^{++} \tilde{p}_{0}^{x 2}\left[\cos \left(\theta_{0}\right)-\cos \left(\theta_{0}\right)-2 \phi_{j}\right]-\lambda_{j} \cos \left(\theta_{j}\right)\left(\tilde{p}_{0}^{x 2}-\frac{2}{\alpha_{\mathrm{PSF}}}\right)-\frac{1}{2}\left(T^{++}-T^{+-}\right) \tilde{p}_{0}^{x 2} \cos \left(-2 \phi_{j}\right), \\
\hbar D_{j k} & =\left\{\begin{array}{l}
-3 \lambda_{j} \cos \left(\theta_{j}\right)-\frac{3}{2}\left(T^{++}+T^{+-}\right), \quad j=k, \\
-2 \lambda_{k} \cos \left(\theta_{k}+2 \phi_{k}-2 \phi_{j}\right)-\left(T^{++}+T^{+-}\right) \cos \left(2 \phi_{k}-2 \phi_{j}\right), \quad j \neq k .
\end{array}\right.
\end{aligned}
$$

Analogously to Ref. [22], we remove the phases as dynamical variables by assuming locked phases and linearization. We define the time-dependent phase as $\phi_{j}(t) \equiv \delta \phi_{j}(t)+\phi_{j}^{(0)}$, where the locked phases satisfy $K_{j}\left(\phi_{j}^{(0)}\right)=0$ and $\delta \phi_{j}(t)$ is a small deviation. Expanding equations (B7) and (B8) up to first order in $\delta \phi_{j}$ around $\phi_{j}^{(0)}$ and neglecting terms $\propto \delta \phi_{j} \tilde{p}_{k}^{y 2}$ leads to an explicit expression for $\delta \phi_{j}$, which can be substituted back to obtain

$$
\partial \tilde{p}_{j}^{y}=\left[L_{j}\left(\phi_{j}^{(0)}\right)+\sum_{k=1}^{2}\left(C_{j k}\left(\phi_{j}^{(0)}, \phi_{k}^{(0)}\right)-\frac{D_{j k}\left(\phi_{j}^{(0)}, \phi_{k}^{(0)}\right) L_{j}^{\prime}\left(\phi_{j}^{(0)}\right)}{K_{j}^{\prime}\left(\phi_{j}^{(0)}\right)}\right) \tilde{p}_{k}^{y 2}\right] \tilde{p}_{j}^{y} .
$$

We rewrite these two equations in a shorter form:

$$
\begin{aligned}
& \partial_{t} \tilde{p}_{1}^{y}=\tilde{\alpha}_{1} \tilde{p}_{1}^{y}-\tilde{\beta}_{1} \tilde{p}_{1}^{y 3}-\tilde{\theta}_{1} \tilde{p}_{2}^{y 2} \tilde{p}_{1}^{y}, \\
& \partial_{t} \tilde{p}_{2}^{y}=\tilde{\alpha}_{2} \tilde{p}_{2}^{y}-\tilde{\beta}_{2} \tilde{p}_{2}^{y 3}-\tilde{\theta}_{2} \tilde{p}_{1}^{y 2} \tilde{p}_{2}^{y} .
\end{aligned}
$$

We replace $\tilde{p}_{j}^{y}$ with a product of a dimensionless quantity $\hat{\tilde{p}}_{j}^{y}$ and a characteristic quantity $\tilde{p}_{j, c}^{y}$ which carries the original dimension, i.e., $\tilde{p}_{j}^{y}=\hat{\tilde{p}}_{j}^{y} \tilde{p}_{j, c}^{y}$. We do the same for the independent time variable $t=\hat{t} t_{c}$, so that the derivative changes to $\frac{\partial \tilde{p}_{j}^{y}}{\partial t}=\frac{\tilde{p}_{j, c}^{y}}{t_{c}} \frac{\partial \hat{\tilde{p}}_{j}^{y}}{\partial \hat{t}}$. The characteristic values are chosen in a way that the corresponding dimensionless quantities are of magnitude 1 . With the definitions $\hat{t} \equiv t$ and $\hat{\tilde{p}}_{j}^{y} \equiv A_{j}$, we can finally write down the population competition model as

$$
\begin{aligned}
& \partial_{t} A_{1}=\alpha_{1} A_{1}-\beta_{1} A_{1}^{3}-\theta_{1} A_{2}^{2} A_{1}, \\
& \partial_{t} A_{2}=\alpha_{2} A_{2}-\beta_{2} A_{2}^{3}-\theta_{2} A_{1}^{2} A_{2}+S,
\end{aligned}
$$

where we have manually added a control parameter $S$ for the $A_{2}$ mode pair.

\section{APPENDIX C: CALCULATION OF THE MODEL PARAMETERS}

The model parameters can be calculated from the physical parameters via equation (B10). Their values, especially the signs, depend on the specific choice of the locked phases. The system's physical behavior observed in the full numerical simulations suggests destabilizing linear terms and stabilizing nonlinear terms in the PC model. Choosing phases satisfying this condition, characteristic values $\tilde{p}_{j, c}^{y}=1 \mu \mathrm{m}^{-1}$ and $t_{c}=$ $1 \mathrm{ps}$, and anisotropy effects $\delta_{1}=0.2 \mathrm{meV}$ and $\delta_{2}=0 \mathrm{meV}$ leads to the following model parameters for the switching simulation presented in Sec. II: $\alpha_{1}=0.49, \alpha_{2}=0.43, \beta_{1}=$ 0.007, $\beta_{2}=0.01, \theta_{1}=0.006, \theta_{2}=0.005$. This corresponds to the case of larger self-saturation. The anisotropy is sufficiently high for the initial pattern formation and back switching, and also no hysteresis can occur. 
[1] X. Hu, P. Jiang, C. Ding, H. Yang, and Q. Gong, Picosecond and low-power all-optical switching based on an organic photonicbandgap microcavity, Nat. Photon. 2, 185 (2008).

[2] A. M. C. Dawes, L. Illing, S. M. Clark, and D. J. Gauthier, Alloptical switching in rubidium vapor, Science 308, 672 (2005).

[3] D. Ballarini, M. De Giorgi, E. Cancellieri, R. Houdré, E. Giacobino, R. Cingolani, A. Bramati, G. Gigli, and D. Sanvitto, All-optical polariton transistor, Nat. Commun. 4, 1778 (2013).

[4] A. Amo, T. Liew, C. Adrados, R. Houdré, E. Giacobino, A. V. Kavokin, and A. Bramati, Exciton-polariton spin switches, Nat. Photon. 4, 361 (2010).

[5] D. Sanvitto and S. Kéna-Cohen, The road towards polaritonic devices, Nat. Mater. 15, 1061 (2016).

[6] R. Kheradmand, M. Sahrai, H. Tajalli, G. Tissoni, and L. A. Lugiato, All optical switching in semiconductor microresonators based on pattern selection, Eur. Phys. J. D 47, 107 (2008).

[7] S. Schumacher, N. H. Kwong, R. Binder, and L. Smirl Arthur, Low intensity directional switching of light in semiconductor microcavities, Phys. Status Solidi RRL 3, 10 (2009).

[8] M. H. Luk, Y. C. Tse, N. H. Kwong, P. T. Leung, P. Lewandowski, R. Binder, and S. Schumacher, Transverse optical instability patterns in semiconductor microcavities: Polariton scattering and low-intensity all-optical switching, Phys. Rev. B 87, 205307 (2013).

[9] P. Lewandowski, S. M. H. Luk, C. K. P. Chan, P. T. Leung, N. H. Kwong, R. Binder, and S. Schumacher, Directional optical switching and transistor functionality using optical parametric oscillation in a spinor polariton fluid, Opt. Express 25, 31056 (2017).

[10] M. C. Cross and P. C. Hohenberg, Pattern formation outside of equilibrium, Rev. Mod. Phys. 65, 851 (1993).

[11] C. Bowman and A. C. Newell, Natural patterns and wavelets, Rev. Mod. Phys. 70, 289 (1998).

[12] H. Meinhardt, Models of Biological Pattern Formation (Academic, London, 1982).

[13] J. D. Murray, Mathematical Biology. II: Spatial Models and Biomedical Applications, 2nd ed. (Springer, New York, 2003).

[14] M. P. Hassell, H. N. Comins, and R. M. May, Spatial structure and chaos in insect population dynamics, Nature (London) 353, 255 (1991).

[15] A. Kavokin, J. J. Baumberg, G. Malpuech, and F. P. Laussy, Microcavities (Oxford University Press, New York, 2008).

[16] V. Ardizzone, P. Lewandowski, M. H. Luk, Y. C. Tse, N. H. Kwong, A. Lücke, M. Abbarchi, E. Baudin, E. Galopin, J. Bloch, A. Lemaitre, P. T. Leung, P. Roussignol, R. Binder, J. Tignon, and S. Schumacher, Formation and control of turing patterns in a coherent quantum fluid, Sci. Rep. 3, 3016 (2013).

[17] O. A. Egorov, A. Werner, T. C. H. Liew, E. A. Ostrovskaya, and F. Lederer, Motion of patterns in polariton quantum fluids with spin-orbit interaction, Phys. Rev. B 89, 235302 (2014).

[18] H. Saito, T. Aioi, and T. Kadokura, Order-Disorder Oscillations in Exciton-Polariton Superfluids, Phys. Rev. Lett. 110, 026401 (2013).

[19] P. Lewandowski, O. Lafont, E. Baudin, C. K. P. Chan, P. T. Leung, S. M. H. Luk, E. Galopin, A. Lemaître, J. Bloch, J. Tignon, P. Roussignol, N. H. Kwong, R. Binder, and S.
Schumacher, Polarization dependence of nonlinear wave mixing of spinor polaritons in semiconductor microcavities, Phys. Rev. B 94, 045308 (2016).

[20] S. Schumacher, Spatial anisotropy of polariton amplification in planar semiconductor microcavities induced by polarization anisotropy, Phys. Rev. B 77, 073302 (2008).

[21] S. Schumacher, N. H. Kwong, and R. Binder, Influence of exciton-exciton correlations on the polarization characteristics of polariton amplification in semiconductor microcavities, Phys. Rev. B 76, 245324 (2007).

[22] Y. C. Tse, C. K. P. Chan, M. H. Luk, N. H. Kwong, P. T. Leung, R. Binder, and S. Schumacher, A population-competition model for analyzing transverse optical patterns including optical control and structural anisotropy, New J. Phys. 17, 083054 (2015).

[23] S. Wiggins, Introduction to Applied Nonlinear Dynamical Systems and Chaos, Texts in Applied Mathematics (Springer, New York, 2006).

[24] L. Brenig, Complete factorisation and analytic solutions of generalized Lotka-Volterra equations, Phys. Lett. A 133, 378 (1988).

[25] J. Hofbauer, K. Sigmund et al., The Theory of Evolution and Dynamical Systems: Mathematical Aspects of Selection (Cambridge University Press, Cambridge, 1988).

[26] B. Hernández-Bermejo and V. Fairén, Lotka-Volterra representation of general nonlinear systems, Math. Biosci. 140, 1 (1997).

[27] P. J. Wangersky, Lotka-Volterra population models, Annu. Rev. Ecol. Syst. 9, 189 (1978).

[28] R. H. Hering, Oscillations in Lotka-Volterra systems of chemical reactions, J. Math. Chem. 5, 197 (1990).

[29] R. M. Goodwin, A growth cycle, in Essays in Economic Dynamics (Macmillan, London, 1982), pp. 165-170.

[30] W. E. Lamb, Jr., Theory of an optical maser, Phys. Rev. 134, A1429 (1964).

[31] J. Roughgarden, Theory of Population Genetics and Evolutionary Ecology: An Introduction (Macmillan, London, 1979).

[32] K. Van Ivanky Saputra, L. van Veen, and G. R. W. Quispel, The saddle-node-transcritical bifurcation in a population model with constant rate harvesting, Discrete Contin. Dyn. Syst. B 14, 233 (2010).

[33] B. Buchberger, Ein Algorithmus zum Auffinden der Basiselemente des Restklassenringes nach einem nulldimensionalen Polynomideal, Ph.D. thesis, University of Innsbruck, 1965.

[34] B. Buchberger, Ein algorithmisches Kriterium für die Lösbarkeit eines algebraischen Gleichungssystems, Aequationes Math. 4, 374 (1970).

[35] M. Scheffer, J. Bascompte, W. A. Brock, V. Brovkin, S. R. Carpenter, V. Dakos, H. Held, E. H. Van Nes, M. Rietkerk, and G. Sugihara, Early-warning signals for critical transitions, Nature (London) 461, 53 (2009).

[36] J. R. Tredicce, G. L. Lippi, P. Mandel, B. Charasse, A. Chevalier, and B. Picqué, Critical slowing down at a bifurcation, Am. J. Phys. 72, 799 (2004).

[37] S. H. Strogatz, Nonlinear Dynamics and Chaos: With Applications to Physics, Biology, Chemistry and Engineering (Westview Press, Cambridge, Massachusetts, 2000), pp. 199, 202. 